\title{
Comparative Analysis of Contrast Enhancement Techniques in Digital Image Processing
}

\author{
Palpinder Kaur \\ Department of Computer Science and Engineering \\ Baba Banda Singh Bahadur Engineering College, \\ Fatehgarh Sahib, Punjab, India
}

\author{
Deepak Aggarwal \\ Professor \\ Department of Computer Science and Engineering \\ Baba Banda Singh Bahadur Engineering College, \\ Fatehgarh Sahib, Punjab, India
}

\begin{abstract}
Contrast enhancement is considered as one of the interesting as well as the visually appealing technique used for enhancement of contrast of an image. It is involved in the area of image processing where several operations such as enhancing contrast, reduction of noise for improved quality of the images are measured. There is a number of contrast enhancement techniques are available and proposed by several researchers. Considering, this paper provided a survey of different contrast enhancement such as HE, BBHE, DSIHE, MMBEBHE, RMSHE, MHE, BPDHE and LCS and Low pass and High pass filtering techniques.
\end{abstract}

\section{General Terms}

Filtering techniques, Contrast, Intensity value.

\section{Keywords}

Image Processing, Contrast Enhancement, Low Pass and High Pass filters.

\section{INTRODUCTION}

Applications that are based on image processing involves an image enhancement process which aims at enhancing images for the purpose of giving satisfactory view to human vision system besides providing a high-quality input to several image processing systems. The quality of an image may get degraded due to distorting imaging systems or lack of skilled operators that are required to handle that image. Another reason for the low-quality image is inappropriate environmental conditions at the time of capturing the image. The quality of the image is improved by removing the noise, manipulating the contrast, applying filters and highlighting the edges in enhancement process. Distinct features in an image can be highlighted and made more visible by adjusting the image contrast which is done in Contrast Enhancement. The difference of lowest and highest intensity values provides the dynamic range of an image by which contrast is obtained. A wide range of applications requires image enhancement techniques in order to obtain high contrast images by manipulating the low contrast ones that are relatively easier to perceive. There are two types of the contrast enhancement algorithms that are:

\section{(i) Frequency domain techniques}

(ii) Spatial domain techniques.

Image pixels are transformed directly under the techniques that deal with the spatial domain. The Fourier transform of the image is manipulated to achieve frequency domain contrast enhancement. Techniques that operate on the basis of frequency domain involve the calculation of Fourier transform of the image which is the process of converting an image into the frequency domain. This process is followed by the application of certain operations on Fourier transform. The inverse of Fourier transform is applied so as to obtain the final output image [3].

The dynamic range of intensity values of pixels is manipulated in order to achieve contrast enhancement. Some systems such as pattern recognition, computer vision, and digital image processing require critically improved images with rich contrasts. Different distortions can lead to degraded quality of images which may get introduced during image acquisition by unskilled photographers or inefficient capturing devices. The luminance level of surrounding in which the image was captured may affect the contrast of an image. The task to interpret and process low contrast image is highly difficult for automated systems that work on digital image processing, pattern recognition and digital printing techniques that requires critical analysis of the input image. Therefore such applications essentially need images with high-quality contrasts [9].

The image is made highly clear so as to enhance its quality which serves as the main objective of Contrast Enhancement. The detection and analysis of the image is a crucial part in medical fields which requires right application of contrast enhancement process. Neighborhood operations are involved in the related techniques that are required to smooth edges and improve the contrast of the image. In order to generate an output pixel, the neighboring pixels over a small area are combined together. The contrast of output image is enhanced through Imadjust. Techniques like Sigmoid and Bilateral retinex function are easy to implement that is defined for all possible real input values so as to achieve enhanced contrast images. Each point has a derivative which is positive. The values of distinct parameters such as RMSE, PSNR, and MSE are computed through the application of various techniques which is followed by the histogram representation of the chosen image. The count of errors is determined by MSE which is defined as a cumulative squared error. Peak signal to noise ratio (PSNR) is calculated by finding the ratio of signal power to the power noise whose higher value indicates the less amount of noise is present. Signal provides the actual data whereas the error is introduced by the noise in this ratio. This indicates that for obtaining higher quality contrast enhancement PSNR must bear higher value while MSE is kept the minimum. The contrast between different techniques is represented by NC while RMSE is given good contrast [10].

\section{CONTRAST ENHANCEMENT TECHNIQUES}

There are several contrast enhancement techniques used for enhancing the contrast while reducing the error rate. Some of them are: 


\subsection{Histogram Equalization (HE)}

Several application fields employ the use of histogram equalization technique to enhance the image contrast. Cumulative density function is used for performing histogram flattening along with the stretching the dynamic range of gray levels. This technique is not preferable for electronic products due to the reason that its level of brightness gets manipulated considerably which may result in unpleasant view of certain objects in the changed contrast and brightness levels.

\subsection{Brightness Bi-Histogram Equalization (BBHE)}

The technique based on $\mathrm{Bi}-\mathrm{HE}$ (BBHE) was presented in order to over the limitation of HE which caused changed brightness levels with modified contrast. The input image is divided into two distinct sub-images. Each sub-image is then manipulated through image mean gray level which is later followed by the application of $\mathrm{HE}$ on each sub-image individually. The level of mean gray-level (mean) value lies in the range of brightness of original image. No insignificant objects are processed or manipulated in this type of hybrid technique which involves both HE methods that retain the brightness of input image and clipped histogram equalization methods.

\subsection{Dualistic Sub Image Histogram Equalization (DSIHE)}

Like in the case of BBHE, dualistic sub-image histogram equalization (DSIHE) partitions the original image into two distinct sub-images. Unlike in BBHE that uses mean gray levels, 0.5 valued cumulative probability density gray levels in histogram are divided distinctively. This means partitioning of the image is carried out with the approach to maximize Shannon's entropy value of resultant image than using mean gray level. The probability of accumulated gray level of input image is decomposed. The two distinct equalized images after being processed are combined together to generate the resultant sub-image histogram that is of dual nature.

\subsection{Minimum Mean Brightness Error Bi- Histogram Equalization (MMBEBHE)}

Minimum Mean Brightness Error Bi-Histogram Equalization is based on the use of the threshold level unlike in the case of BBHE and DSIHE. The subdivision of images into two independent images is determined by this threshold level. Through this difference between the mean gray level (brightness) between the original and resultant image can be minimized to a considerable level. This technique overcomes the problem of previous methods that performed the partitioning of an image into two sub-images that were entirely based on the original input image.

This process of decomposition is followed by the equalization of the histograms in order to obtain the resultant image by the application of HE method. The generation of the resultant image corresponding to each threshold level is not involved in this instead brightness of the resultant image can be achieved

directly. Therefore it is highly efficient for the applications in which time is a critical aspect. Certain assumptions are made while working with this approach. Three steps are followed in MMBEBHE techniques that are mentioned below:

1. AMBE value corresponding to each threshold level is evaluated.

2. Locate the threshold level, XT that bears the least MBE value.
3. The two distinct sub-images which were decomposed on the basis of obtained XT are equalized individually by BBHE technique.

\subsection{Recursive Mean Separate Histogram Equalization (RMSHE)}

Unlike the methods discussed previously which involved single decomposition of the original image, it is decomposed recursively under Recursive Mean Separate Histogram Equalization (RMSHE).A number of sub-images equal to $2 r$ are generated recursively up to the scale of $r$ when the $r=0$ and $\mathrm{r}=1$ respectively. Histogram Equalization technique is then applied to the obtained sub-images distinctively. RMSHE is similar to HE method when no sub-image is generated i.e. $r=1$ and it corresponds to BBHE technique when the value of $r$ is equal to 1 . With the rise in the value of $r$ that represents the distinction levels the preservation value of the resultant image also increases.

The successive decomposition of the new histograms is accomplished that is determined by their mean value in order to achieve recursive image decomposition. The increasing recursive mean separation levels in the output image results in a convergence of brightness of output image to the brightness of input image which is indicated by mathematical analysis. This technique is applicable to the electronic devices that require scalability in brightness preservation levels which is offered by RMSHE.

\subsection{Multi Histogram Equalization (MHE)}

Multi Histogram Equalization (MHE) provides a more realistic look at the resultant image which is achieved through multiple separations of the actual image into various distinct sub-images. This is followed by the equalization of the histograms that offers enhanced contrast in the modified image. The entire image separation process into sub-images is determined by the histogram of the input image whose threshold levels classifies them into different classes. Each sub-image is given by individual sub-images. Multiple thresholds are chosen to partition the original image into distinct sub-images in the segmentation phase of digital image process. The technique or the method used to perform the subdivision of the input image determines the count of the subimages it form [8].

This entire technique is based on the steps that are mentioned below:

1. Decomposing the histogram several times.

2. Appropriate thresholds are selected.

3. Automatic thresholding criterion.

\subsection{Brightness Preserving Dynamic Histogram Equalization (BPDHE)}

This technique considers the local maxima to achieve decomposition of input image into numerous distinct subimages. Here, individual histogram is equalized dynamically to form the resultant image by joining the sub-images. The mean intensity value is retained in the obtained resultant image as it was in the original input image. This helps in maintaining the brightness levels after the application of this technique. Prior to the decomposition of the histograms on the basis of local maxima single dimensional Gaussian filter is applied to smoothen the histograms of the input image. Each partition of the histogram is associated with a new dynamic range which required in achieving equalized histograms. Normalization of the resultant image is performed to obtain similar brightness as that of an original image. BPDHE involves the application of the steps given below: 
1. Application of Gaussian filters to smooth the histograms.

2. Local maxima are located among the smoothed histogram.

3. The new dynamic range is associated to histogram partitions individually.

4. Histogram equalization is performed on every partition.

5. Image brightness is normalized.

\subsection{Local and Global Contrast Stretching (LCS)}

To enhance the image different elements are manipulated in LCS to improve the view of the entire image comprising of both dark and light portions through operations performed simultaneously. The element located at the center is manipulated and windows slide across the entire input image. Adjustment of the center element is done on the basis of the equation given below:

$$
I_{p}(x, y)=255 * \frac{\left[I_{o}(x, y)-\min \right]}{\max -\min }
$$

where,

The resultant color level is of output pixel at $(\mathrm{x}, \mathrm{y})$ of after contrast stretching is given by $I_{p}(x, y)$

The color level input for data of the pixel(x,y) is represented by $I_{o}(x, y)$

The maximum value for the color level in the input image is given by $\max$.

The minimum value for the color level in the input image is given by min.

The $(\mathrm{x}, \mathrm{y})$ indicates the position of the center element of the input image in KERNEL in the equation mentioned above. In the chosen KERNEL the minimum and maximum value for the original input are given by $\min$ and max respectively. Every color palate range in the original image is considered in the local contrast stretching process. Variety of color range of input image is presented for the purpose of contrast stretching. For contrast stretching, each color palate is assigned with a set of values that contains minimum and maximum values. To achieve Global contrast stretching every single color palate range is considered. For RGB color only a single value will be returned for minimum and maximum respectively on the combination of distinct RGB colors which will be used up in the process of contrast stretching [7].

\section{FILTERING TECHNIQUES}

Image enhancement process involves the enhancing the input image in a way to give it a new improved look for better understanding of the image by both automated systems and humans. Noise or certain distortions in the original image can be eliminated by performing the filtering process including Fourier filtering so as to highlight prominent image features that focus on high, low or directional spatial frequency. An image can be enhanced in terms of spatial domain or in frequency domain. Such techniques are different for obtaining smoothened and sharpened images.

\subsection{Low-pass filter (smoothing)}

Signals that have low-frequency are allowed to pass through low-pass filters that attenuate frequency signals that have value greater than that of the cut-off value. The attenuation level for individual frequency is determined by the design of a filter. Low-pass filtering provides the smoothing of the original image in the frequency domain [1] [6] [2]. Both Gaussian and Butterworth filters are ideal low-pass filters that are able to provide standardized smoothed images.

\subsubsection{Ideal Low-pass Filters}

Nearly all the components in Fourier Transformation that corresponds to high frequency are removed by such filters which are distant from the Transform origin with distance greater than the mentioned distance D [7].

The transfer function of an ideal Low-pass filter is given below:

$$
H(u, v)=\left\{\begin{array}{l}
1 \text { if } D(u, v) \leq D 0 \\
0 \text { if } D(u, v)>D 0
\end{array}\right.
$$

Here,

$\mathrm{D}(\mathrm{u}, \mathrm{v})$ represents the distance from point located at $(\mathrm{u}, \mathrm{v})$ to the center of their frequency.

$$
D(u, v)=\left[\left(u-\frac{M}{2}\right)^{2}+\left(V-\frac{N}{2}\right)^{2}\right]^{1 / 2}
$$

\subsubsection{Butterworth Low-pass Filters}

Noise with high frequency is eliminated from the signal by using frequency-domain filter which causes minimum loss of signal data in the order $\mathrm{n}$ pass-band which is given below:

$H(u, v)=\frac{1}{1+\left[\frac{D(u, v)}{D 0}\right]^{2 n}}$

\subsubsection{Gaussian Low-pass Filters}

Gaussian Low-pass filter has the transfer function that is defined by the equation mentioned below:

$$
H(u, v)=e^{-D^{2}(u, v) / 2 D_{0}^{2}}
$$

\subsection{High-pass filters (sharpening)}

High frequency components are allowed to pass through highpass filters and provides for the attenuation of frequencies that are below the level of cut-off frequency [11]. High-pass filtering is performed in the frequency domain to achieve image sharpening. Gaussian and Butterworth are the ideal high-pass filters that are commonly used for image smoothing process. Generally, high-pass filters are related to low-pass filters through the equation given below:

$$
H h p=1-H l p
$$

\section{LITERATURE SURVEY}

Several researchers have proposed different techniques to enhance the contrast of an image while remaining its original quality. Some work of the researchers is shown as:

Anureet Kaur, "Region of Interest based Contrast Enhancement Techniques for CT images", [1] presented a region extraction algorithm that focused on level set evolution. Enhanced quality of image was achieved through this technique as it reduced the noise in the signal. CT images were processed to enhance the contrast that essentially required un-sharp mask filtering. The comparison between the existing techniques with the presented work indicated the higher efficiency level of this technique.

Akshay Girdhar, "Region Based Adaptive Contrast Enhancement of Medical Ultrasound Images", [2] proposed the adaptive technique for enhancing contrast that focused upon region growing segmentation. Seed point of the desired portion was chosen automatically. Segmentation of both background and foreground pixels was achieved through the processing of thresholds. The foreground was enhanced specifically so as to enhance the prominent features necessarily. The proposed work offered higher performance with better results than the techniques involving state-of-the art approach.

A. Djerouni, "MR imaging contrast enhancement and segmentation using fuzzy clustering", [3] presented use of 
Fuzzy C-Means (FCM) algorithm that was employed for segmenting the medical images which required local image contrast enhancement preprocessing. The originality of the adaptive of convolution mask coefficients was provided by this convolution filtering. This used equation derived by the Newtonian model to locate the distribution of gray-level of neighboring pixels that was considered to be $1 / r^{2}$ where $r$ represented the hybrid distance. The comparative results showed the better output was given by this technique. The grey level distribution of pixels in the neighborhood of the current pixel is considered as $1 / \mathrm{r} 2$ distribution, which was deduced from the Newtonian model, where $r$ is a hybrid distance which involves the spatial information and the luminance one. Finally, some results are presented in order to show the computational performance of this approach.

Chin Yeow Wong, "Image contrast enhancement using histogram equalization with maximum intensity coverage", [5] proposed the technique in order to maximize the content that yielded significant information by enhancing the color of image and hinders the loss of data by insignificant objects. The obtained intermediate equalized image was weighed against original image cover entire intensity range was covered. The application of golden section search algorithm provided resultant weighing factor. The presented technique was more efficient in recovering information from images that were captured in all possible environments as compared other techniques. It was highly capable of providing resultant sharp images with improved contrast.

Navdeep Kanwal, "Region Based Adaptive Contrast Enhancement of Medical X-Ray Images", [7] proposed a contrast enhancement technique with an adaptive neighborhood approach that involved hybrid methods for processing X-ray images essentially. Experimental results showed the technique of the proposed work to be relatively better than other existing ones.

Namita Naik, "Low Contrast Image Enhancement using Wavelet Transform based Algorithms: A Literature Review" [8] presented the survey regarding techniques that operated on the algorithms that were combined with wavelet transform like discrete wavelet transform (DWT) and Undecimated Wavelet Transform(UWT) which suggested the UWT as an emerging research fields due to its higher efficiency levels.

Ritika, "Contrast Enhancement Techniques for Images- A Visual Analysis", [9] presented the issue of low contrast images by analyzing the mathematical morphological technique and showed the comparison between the proposed technique with other existing approaches. Despite certain limitations offered by the Histogram Equalization (HE) technique, it was capable of enhancing the image contrast globally. The local contrast of the image could be improved through the implication of Contrast Limited Adaptive Histogram Equalization (CLAHE). This did not cause any noise amplification. White and black top-hat is employed in Morphological-contrast enhancement which could be applied to both single and multiple scales of structures that could of varying shapes or sizes.

Vijay A. Kotkar, "Review Of Various Image Contrast Enhancement Techniques", [11] studied certain techniques that were used for improving image contrast but the application of those techniques caused changed brightness level of image which served as the major concern. Hence, a technique that involved the combination of both local and global contrast enhancement might provide retained brightness. Several existing techniques like HE, BBHE, DSIHE etc. for contrast improvement were reviewed with comparative approach that entirely focused on the local and global enhancement techniques. Applications like image processing required in industrial X-ray, hyper spectral, medical image analysis, remote sensing used the proposed technique.

After having a review to the previous work in the field of image contrast enhancement, it is observed that there are some issues that are questionable or have not been solved yet. These issues are:

a. As noise is one of the major factors that affect the quality of an image and there are various types of noises that have different impact on images. Widely, the focus of the research works was on additive noise, salt and pepper noise and multiplicative noise. Whereas the effect of noise likes Gaussian noise was not observed much.

b. It has been observed that mostly traditional filtration techniques were used that were not much effective.

c. Recent image contrast enhancement techniques were quite effective to improve the contrast of the images but it degrades the brightness of the image which directly leads to the reduction in quality of the image.

The above defined research challenges are need to be solved by using advance image contrast enhancement techniques along with the prominent and effective filtration techniques. Moreover, The common parameters that are used in traditional image contrast enhancement techniques are entropy, standard deviation and Contrast to Noise ratio (CNR).

\section{CONCLUSION AND FUTURE SCOPE}

It has been seen that a large number of authors have been conducted their research work in the domain of image processing to improve the existing techniques so that the informative part of the images can be utilized properly. Image processing is such a wide field and covers a large number of topics in it such as image segmentation, image enhancement, image contrast enhancement etc. This paper has provided a survey of several contrast enhancement techniques with their basic properties. The techniques like HE, BBHE, DSIHE, MMBEBHE, RMSHE, MHE, BPDHE and LCS are reviewed. Moreover, the applicability of these techniques in numerous domains is also discussed in this paper. Additionally, filters have been introduced which are used to remove noise from an image while enhancing its quality. Basically, low pass and high pass filters are employed for filtering.

In future, advanced filters like Kuwahara to remove the noise from the image and along with this advanced region of interest based mechanism can be applied to perform the contrast enhancement only to the informative portion of the image without affecting rest of the image.

\section{REFERENCES}

[1] Kaur, A., Girdhar, A., \& Kanwal, N. (2016, February). The region of interest based contrast enhancement techniques for CT images. In Computational Intelligence \& Communication Technology (CICT), 2016 Second International Conference on(pp. 60-63). IEEE.

[2] Girdhar, A., Gupta, S., \& Bhullar, J. (2015, February). Region-Based Adaptive Contrast Enhancement of Medical Ultrasound Images. In Computational Intelligence \& Communication Technology (CICT), 2015 IEEE International Conference on(pp. 750-753). IEEE. 
[3] Djerouni, A., Hamada, H., \& Berrached, N. (2011). MR imaging contrast enhancement and segmentation using fuzzy clustering. IJCSI International Journal of Computer Science Issues, 8(4).

[4] Makandar, A., \& Halalli, B. (2015). Image enhancement techniques using highpass and lowpass filters. International Journal of Computer Applications, 109(14)

[5] Wong, C. Y., Liu, S., Liu, S. C., Rahman, M. A., Lin, S. C. F., Jiang, G., ... \& Shi, H. (2016). Image contrast enhancement using histogram equalization with maximum intensity coverage. Journal of Modern Optics, 63(16), 1618-1629.

[6] Cao, G., Huang, L., Tian, H., Huang, X., Wang, Y., \& Zhi, R. (2017). Contrast enhancement of brightnessdistorted images by improved adaptive gamma correction. Computers \& Electrical Engineering.

[7] Kanwal, N., Girdhar, A., \& Gupta, S. (2011, May). Region-based adaptive contrast enhancement of medical
X-ray images. In Bioinformatics and Biomedical Engineering,(iCBBE) 2011 5th International Conference on (pp. 1-5). IEEE.

[8] Naik, N., \& Mishra, A. (2015). Low contrast image enhancement using wavelet transform based algorithms: a literature review. Int. J. Eng. Tech. Res, 3(6), 123-128.

[9] Kaur, S. (2013). Contrast enhancement techniques for images-A visual analysis. International Journal of Computer Applications, 64(17).

[10] Kaur, R., \& Kaur, S. (2016, March). Comparison of contrast enhancement techniques for a medical image. In Emerging Devices and Smart Systems (ICEDSS), Conference on (pp. 155-159). IEEE.

[11] Kotkar, V. A., \& Gharde, S. S. (2013). Review of various image contrast enhancement techniques. International journal of innovative research in Science, Engineering, and Technology, 2(7). 